\title{
Comparison of Pressure and Intensity Methods in Evaluating the Directional Diffusion Coefficient
}

\author{
A. Pilch*, T. Kamisiński And M. Zastawnik \\ AGH - University of Science and Technology, Faculty of Mechanical Engineering and Robotics \\ Department of Mechanics and Vibroacoustics, al. A. Mickiewicza 30, 30-059 Krakow, Poland
}

\begin{abstract}
The directional diffusion coefficient characterizes directional uniformity of acoustic energy reflected from a structure. The goal of the paper is to check whether different measurement methods of that coefficient give comparable results and can be used for different diffusing structures. ISO 17497-2:2012 recommends two basic measurement methods for this parameter, both based on sound pressure analysis. In the first method, one microphone and a measurement manipulator is used (the space method), while in the second one, 19 microphones placed on the sound-reflecting plane are required (the boundary method). In the standard it is assumed (as usually in the room acoustics), that the acoustic energy is proportional to the square of sound pressure, what is true only for the plane wave. Correctness of this assumption was checked by the modified space method where the sound intensity probe was installed instead of microphone. The test revealed that pressure methods gave comparable results for both low- and high-diffusion structures, with the boundary method giving moderately higher values for low-diffusion structures and slightly higher for high-diffusion structures. The results obtained in the intensity method were comparable with the pressure method except for the $2000 \mathrm{~Hz}$ frequency range.
\end{abstract}

DOI: $10.12693 /$ APhysPolA.123.1054

PACS: 43.55.-n, 43.55.Ka, 43.55.Mc, 43.20.Ei

\section{Introduction}

Sound absorption is the most important property of materials used in acoustical treatment of interiors. It is well defined and widely used by architects and acousticians to predict reverberation time and other acoustic parameters of rooms. There are many formulas based both on theory and experiment used to calculate reverberation time just from geometry of a room and sound absorption of materials used there. Beranek [1] was the first to draw attention to diffusion properties of surfaces making a room and their effect on its acoustics. But the parameter defined by him and known as the surface diffusivity index (SDI) was obtained on the grounds of visual inspection of interiors, therefore it was subjective and impossible to be built-in in any quantitative theory of the reverberation time. The fact that reflections from walls are not purely specular and its consequence for acoustics properties of rooms was included in reverberation time formulae by Kuttruff in 1976 [2]. The diffusion could be taken into account more precisely in calculations based on the ray tracing method and the image source method. Comparative tests of simulation computer programs used for determination of room acoustics parameters have revealed that only those tools in which the sound diffusion effect was taken into account were capable to predict results close to values obtained in experiments [3]. In 1975 Schroeder has laid the foundations for designing and measuring high-scattering structures for the purposes of room

*corresponding author; e-mail: apilch@agh.edu.pl acoustics [4]. He was the first to present an approximate formula for distribution of reflected sound $S(\alpha)$ from a structure with variable reflection coefficient $r(x)$ :

$$
S(\alpha)=\int r(x) \exp \left[2 \pi j x\left(\sin \alpha-\sin \alpha_{i}\right) / \lambda\right] \mathrm{d} x,
$$

where $\alpha$ is the receiver angle, $\alpha_{i}$ is the angle of incidence, and $\lambda$ is the wavelength.

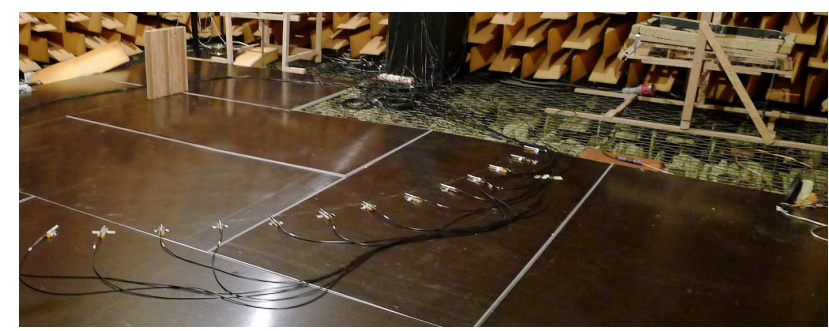

Fig. 1. A measurement set-up for determining the directional diffusion coefficient by means of the boundary method.

Schroeder has obtained the directional characteristics of the reflected wave field using $0.03 \mathrm{~m}$ electromagnetic waves. The first measurements with the use of acoustic waves were performed only in the 1990s when a dynamic development of measurement and calculation methods occurred in the area of sound diffraction and key quantities defining the related phenomena were defined.

The directional sound diffusion coefficient (usually denoted $d$ ) is a measure of quality of the directivity characteristics of sound waves reflected from a structure. Determination of energy distribution uniformity for different reflection angles was based initially on calculation of 
the standard deviation [5], directivity [6], or the specular reflection zone [7]. Presently, uniformity of a directional characteristics is determined usually by means of the autocorrelation function [8]:

$$
d_{0}=\frac{\sum_{i} \sum_{j \neq i} I_{i} I_{j}}{(n-1) \sum_{i} I_{i}^{2}},
$$

where $I_{i}$ is the scattered intensity, measured at $n$ different angles. In ISO 17497-2 standard [9] the intensity is represented by the squared acoustic pressure level $L_{i}$ expressed in logarithmic (decibel) scale and the following formula is used [9]:

$$
d_{0}=\frac{\left(\sum_{i=1}^{n} 10^{L i / 10}\right)^{2}-\sum_{i=1}^{n}\left(10^{L i / 10}\right)^{2}}{(n-1) \sum_{i=1}^{n}\left(10^{L i / 10}\right)^{2}} .
$$

Sound scattering on test sample edges with determined dimensions increases with decreasing frequency - for sufficiently long waves, a surface with finite dimensions will act as a point source emitting diffused sound in all directions. To eliminate the effect of sound scattering occuring on the sample edge on diffusion measurement results, the normalized directional diffusion coefficient was introduced as a quantity calculated with taking into account the diffusion coefficient $d_{\mathrm{r}}$ determined for a flat soundreflecting plate with dimensions identical with those of the examined sample [10]:

$$
d=\frac{d_{0}-d_{\mathrm{r}}}{1-d_{\mathrm{r}}}
$$

For low frequencies or for a sample showing very low sound diffusion, as well as for a concave element focusing sound at a position occupied by one of the receivers, the normalized directional diffusion coefficient can be less then 0 . In such cases, ISO 17497-2 standard [9] recommends that the coefficient value is peremptorily assumed to be zero.

The directional sound diffusion coefficient is widely used in design work on solutions to be applied where undesirable phenomena are observed in regions close to the diffusing structures. This is of special interest in describing diffusers located in small rooms or placed close to listeners, where local irregularities of sound reflections can significantly deteriorate the sound quality.

Measurement of the directional sound diffusion coefficient involves determination of the impulse response function in a number of points for three configurations of the measurement set-up. Measurements are taken for the sample, the reference plate and the set-up without any test object. This way, it is possible to eliminate the effect of disturbing sound reflections coming from the setup's equipment. It is therefore very important to maintain positions of the microphone as precisely as possible in the subsequent experiments. This was achieved by positioning the microphone in space with the use of specialist measurement manipulator (hereinafter referred to as "the space method"). It is important that the ma- nipulator has a structure minimizing the effect of sound reflections and allowing to displace a microphone over surface of a hemisphere with a definite radius [11]. Measurement require moreover an anechoic chamber without sound-reflecting floor [12].

An alternative to this method was proposed by D'Antonio who has located the sample, the sound source, and measurement microphones on a sound-reflecting plane ("the boundary method") [13]. The method does require neither manipulator nor anechoic chamber. What is needed is a set of microphones located on flat surface in a large room. The number of receivers depends on assumed angular resolution of the measurement. This means that $n=90^{\circ} / \delta+1$ microphones are needed distributed evenly along a quarter of a circle and separated by an angle $\delta$; typically, $\delta=5^{\circ}$ and $n=19$.

In the boundary method, acoustically hard surface acts as a mirror for the sound-diffusing structure creating the image source on the other side of the surface. In other words, measured is a diffuser and its mirror image. It is therefore necessary to locate both the sound source and receiver as close as possible to the reflecting surface as possible (Fig. 1). If $r_{1}$ is the distance between the sound source and the measured sample, $r_{2}$ is the distance from the point of intersection of the sample with the reflecting surface to the microphone, and $r_{3}$ is the distance from the same point to the sound source, then the measurement upper limiting frequency $f_{g}$ is given by the formula

$$
f_{g}=\frac{c}{2\left|r_{1}-r_{2}-r_{3}\right|},
$$

where $c$ is the speed of sound.

Microphones should be placed on thin elastic pads in view of the possibility of sound transfer via the reflecting surface material leading potentially to increase of the overall measurement error.

Determination of the averaged directional diffusion coefficient would involve time-consuming and tedious experimental procedure. Vorländer and Mommertz in [14] have proposed a method of measuring diffusion properties of a structure in the scattered field, defining the randomincidence scattering coefficient (usually denoted $s$ ) as the ratio of energy reflected in the non-specular direction and the total acoustic energy reflected from a structure. The quantity is determined in conditions close to those prevailing in actual rooms and for that reason the coefficient is commonly used in dedicated room-acoustics calculation algorithms. The random-incidence scattering coefficient was defined in ISO 17497-1 standard in the year 2004 [15], however, studies on the appropriate measurement procedure are still continued [16].

An inconvenience in using the $s$ coefficient in practice consists in difficulties with predictions as for expected values of the parameter and the fact that for the measurement, a circular sample with diameter of at least $3 \mathrm{~m}$ is required in order to meet diffraction criteria when measurements are to be started from $100 \mathrm{~Hz}$ octave band as recommended by standards. The parameter is little sensitive to non-uniformity of the reflection directional char- 
acteristics - e.g. redirection of sound out of the specular direction is interpreted as a scattering of sound. On the other hand, its unquestionable advantage consists in the fact that when measuring the random-incidence scattering coefficient $s$, one obtains also the sound absorption coefficient $\alpha$ that, especially for large rooms, is in many cases the parameter more important than the diffusion coefficient itself [17].

This paper is focused on comparison of the pressure (space and boundary) and intensity methods which are used to determine the directional diffusion coefficient for three measured samples with different diffusion characteristics. Up to date, any comparison of pressure methods and the same sample has not been published proving that the two techniques are equivalent. Also, using the sound intensity probe in that kind of measurement has not been reported. It is worth noting that the directional diffusion coefficient is a quantity adopted only recently (the relevant international standard was published in 2012), therefore laboratories testing sound-diffusing structures with the use of this quantity should make their contributions to verification and possible revision of guidelines contained in the standard.

\section{Experimental comparison of the methods}

For comparative measurements, three samples with dimensions $0.45 \mathrm{~m} \times 0.55 \mathrm{~m}$ were used as shown in Fig. 2 .

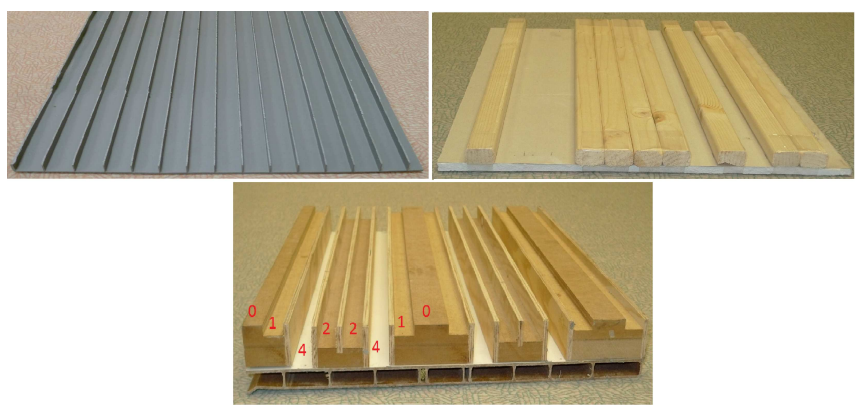

Fig. 2. Structures used to compare the space method and the boundary method. CDW - wells with constant depth; MLS - wells with maximum length sequencebased depths, QRD - with well depths determined by the quadratic residue series.

All samples had a structure of parallel hollows extending over the whole of their length (hereinafter referred to as wells). The depth of the wells in the sample denoted as CDW was constant and equaled $0.02 \mathrm{~m}$. For the MLS sample, the depth of wells was also $0.02 \mathrm{~m}$, while their widths were determined by consecutive terms of a maximum length sequence (MLS). Wells of the structure denoted as QRD had different depths varying from $0.011 \mathrm{~m}$ to $0.044 \mathrm{~m}$ and the same width of $0.022 \mathrm{~m}$. Depths of successive wells corresponded to terms of the quadratic residue for the prime $N=7$.

The measurements were carried out in anechoic chamber of the Department of Mechanics and Vibroacoustics
University at the AGH Univeristy of Science and Technology in Kraków, Poland. The facility is equipped with a measurement manipulator allowing to position the microphone along an arc with radius of $2 \mathrm{~m}$. The mechatronic part of the set-up was controlled by and data acquisition based on the LabView software by National Instruments. Measurements with the use of the boundary method were carried out in MATLAB environment. For all methods, measurements were taken with the acoustic transducers (GRAS 46AE 1/2" microphones in pressure method and B\&K 3520 probe with $0.006 \mathrm{~m}$ microphones spacer in the sound intensity method), situated at a distance of $2 \mathrm{~m}$ from the sample, with the sound source located at the distance of $4 \mathrm{~m}$. As a measurement input signal, exponentially swept sine was used composed of $2^{17}-1$ samples that at the sampling frequency of $96 \mathrm{kHz}$ resulted in a $1.36 \mathrm{~s}$ long signal. In all methods, the impulse response function was determined within the angular range of $90^{\circ}$ with resolution of $5^{\circ}$. The measurements with the use of the pressure space method were performed twice - in November 2009 and in September 2012, with the same geometry maintained in both experiments. However, a different sound source was used and other configuration was adopted for sound-absorbing elements protecting sample edges. It should be noted that measurements of the year 2009 (denoted with suffix _old on the graphs) were carried out on the set-up in the course of its calibration. The plots show both sets of the results in order to illustrate the scale of possible discrepancies between results obtained from measurements carried out with the use of different realizations of the same set-up.

\section{Measurement results}

Results for the structure with constant depth width (CDW) are presented in Fig. 3. The negative values are retained for better illustration of the difference in the directional diffusion coefficient values obtained with different measurement methods.

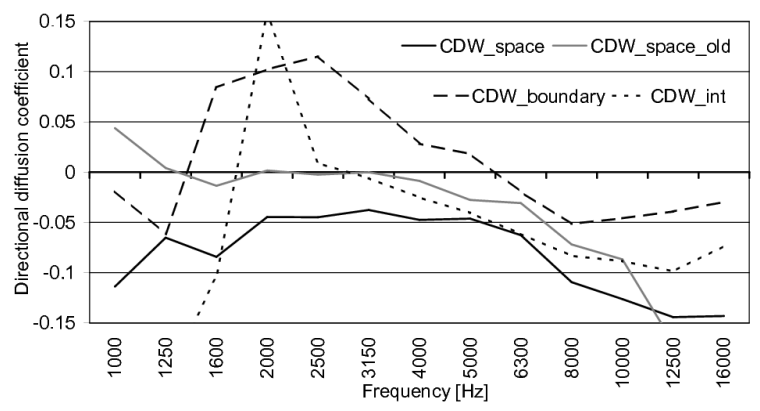

Fig. 3. The directional diffusion coefficient for CDW diffuser measured by means of: CDW space the pressure space method (with suffix _old — the 2009 measurement); CDW_boundary — the boundary method; CDW_int - the intensity space method.

The values obtained by means of the boundary method are much higher than those recorded with the pressure 


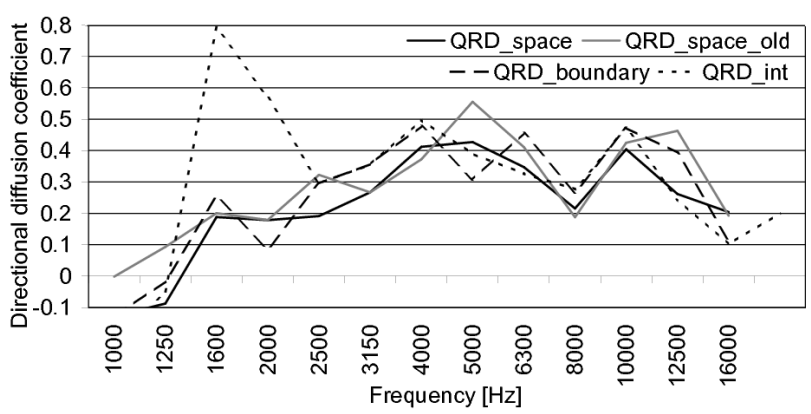

Fig. 4. The directional diffusion coefficient for QRD diffuser measured by means of: QRD_space - the pressure space method (with suffix_old — the 2009 measurement); QRD_boundary — the boundary method; QRD_int — the intensity space method.

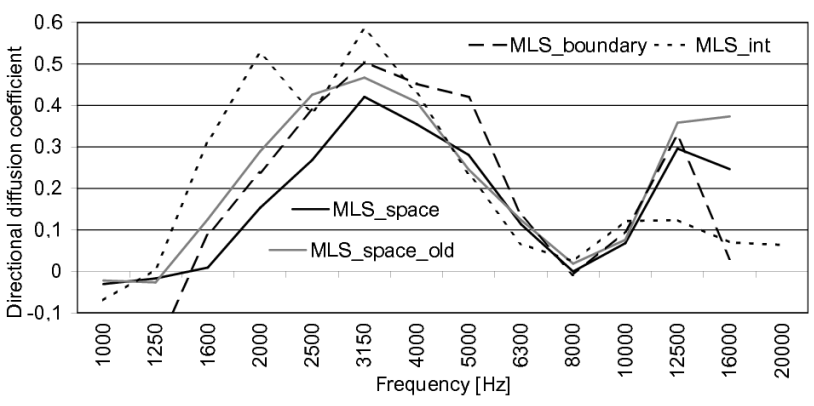

Fig. 5. The directional diffusion coefficient for MLS diffuser, measured by means of: MLS_space the pressure space method (with suffix old - the 2009 measurement); MLS_boundary — the boundary method; MLS_int - the intensity space method.

space method. For the middle range of frequencies, values exceeding 0.1 were obtained in the measurement taken by means of the boundary method, while for the pressure space method, the values were lower than zero for most of the frequency bands. The CDW sample was made of thin plastic and different positioning of the structure (horizontal in the space method and vertical in the boundary method) could result in a slight change of its geometry and/or excitation to different vibration modes. Susceptibility of such sample could also result in an increase of its acoustic absorption. Results of measurements using the sound intensity probe were similar to those obtained with the pressure space method except for $2000 \mathrm{~Hz}$ frequency band, where the intensity method gave surprisingly high value (0.16), while results for space pressure method were -0.05 and 0.00 .

For QRD structure, results are presented in Fig. 4. Likewise as for CDW, measurements performed by means of the boundary method resulted in higher values. The root mean square difference in the range $1-16 \mathrm{kHz}$ between results obtained by means of the two pressure methods was equal to 0.09. The largest dispersion of results was observed for $5 \mathrm{kHz}$ justifying the expectation that this very frequency range is exceptionally sen- sitive to measurement errors for this sample type. A local sound diffusion minimum was identified for all measurements in the $8 \mathrm{kHz}$ frequency band taking the value of 0.19 for the pressure space method and 0.27 for the boundary method. For the frequencies above $2500 \mathrm{~Hz}$, results obtained by means of both methods were very similar. For CDW sample, directional diffusion coefficient values for frequencies near $2000 \mathrm{~Hz}$ were much higher for intensity method. Directional diffusion coefficient for $1600 \mathrm{~Hz}$ exceeded 0.75 , while the design frequency (the lowest freqency for which diffuser can reflect sound in non specular direction) for that type of structure with given well depth was equal to $2234 \mathrm{~Hz}$. Taking into account that the intensity method gave much higher values of diffusion that the pressure methods for all samples for $2000 \mathrm{~Hz}$ frequency band, it can be assumed that the results of the intensity method were not precise for that frequency range.

The best consistency of pressure methods was obtained for MLS structure and frequencies above $5 \mathrm{kHz}$, as shown in Fig. 5. Also in the case of MLS structure, measurement taken with the use of the boundary method resulted in overstated sound diffusion values - especially for frequencies in $1.6-5 \mathrm{kHz}$ range. The intensity method again resulted in much higher values of diffusion for $2000 \mathrm{~Hz}$. What is more, due to $0.006 \mathrm{~m}$ microphone spacer, upper frequency limit of sound intensity probe was about $10000 \mathrm{~Hz}$, so values obtained above limit are not reliable, what results in discrepancies for that frequency range. Significant reduction of diffusion at $8 \mathrm{kHz}$ could be observed in all measurements. Detection of such local decrease in the directional diffusion coefficient values is important in design work on sound diffusing features planned to be applied in actual rooms. Use of MLS structures showing insufficient sound diffusion in the useful frequency band, could result in serious acoustic flaws.

\section{Summary}

Values of the directional diffusion coefficient obtained from tests by means of the boundary method were higher than those measured with both space methods. This may follow from the specular effect that doubled the height of the measured structures and thus affected measurement results despite condition (5) was met. On the other hand, it was found that local minima of the directional diffusion coefficient were correctly located with high accuracy for both QRD- and MLS-type sound-diffusing samples. This allows to use both pressure methods proposed by standard for practical examination of such structures. However, intensity methods, although more precise theoretically (without assumption that the wave reflected from measured structure is plane), results in overestimated values for $2000 \mathrm{~Hz}$ frequency range. Intensity method requires more studies to reveal the origin of that error. Future studies with not only pp (pressure-pressure), but also with pv (pressure-velocity) intensity probe will surely find solution to that problem. 
To apply the boundary method, 19 microphones located on a sound-reflecting plane are typically needed, compared to only one microphone required in the pressure space method. Instead a measurement manipulator for repeatable positioning of the microphone in space on an arc with determined radius is necessary. The measurement as such carried out with the use of the space method will take more time, while preparation of measurement set-up for the boundary method will be more labour- and time-consuming. When designing the setup for the directional diffusion coefficient measurements and selecting the measurement method, the number of samples to be measured must be taken into account. If measurements are planned to be carried out sporadically and an anechoic chamber is available, solution with the use of the measurement manipulator will be more favorable. Such set-up can be also used for a wide spectrum of accurate sound source directivity tests [18]. The space method can be therefore recommended for research laboratories, while the boundary method seems to be more suitable for tests carried out in industrial establishments dealing with production of sound-diffusing structures.

\section{Acknowledgments}

This paper was written with the support of the Faculty Dean's Grant No. 15.11.130.210.

\section{References}

[1] L. Beranek, Music, acoustics $\&$ architecture, Willey, New York 1962.

[2] H. Kuttruff, Acustica 35, 215 (1976).

[3] M. Vorländer, in: Proc. of the 15th ICA, Norway II, 689 (1995).
[4] M. Schroeder, J. Acoust. Soc. Amer. 57, 149 (1975).

[5] T.J. Cox, J. Acoust. Soc. Amer. 97, 2928 (1995).

[6] J. Angus, A.C. Marvin, J. Clegg, J.F. Dawson, in: Proc. 98th Convention AES, Paris 1995, Paper 3955.

[7] T.J. Cox, in 99th Convention AES, New York 1995, Paper 4115.

[8] T. J. Hargreaves, T. J. Cox, Y. W. Lam, P. D'Antonio, in: Proc. 16th International Congress on Acoustics and 135th Meeting Acoustical Society of America, Seattle 1998, Paper 2731.

[9] ISO 17497-2: Acoustics - Sound scattering properties of surfaces - Part 2: Measurement of the directional diffusion coefficient in a free field.

[10] T.J. Cox, P. D'Antonio, Acoustic absorbers and diffusers, Taylor and Francis, London 2009.

[11] J. Felis, A. Flach, T. Kamisiński, Arch. Acoust. 37, 245 (2012).

[12] T.J. Cox, Ph.D. thesis, University of Salford, Manchester 1992.

[13] P. D'Antonio, in Proc. Noise-Con 93, 259 (1993).

[14] M. Vorländer, E. Mommertz, Applied Acoustics 60, 187 (2000).

[15] ISO 17497-1: Acoustics - Sound scattering properties of surfaces - Part 1: Measurement of the random-incidence scattering coefficient in a reverberation room.

[16] T. Kamisiński, K. Brawata, A. Pilch, J. Rubacha, M. Zastawnik, Archives of Acoustics 37, 405 (2012).

[17] T. Kamisiński, K. Brawata, A. Pilch, J. Rubacha, M. Zastawnik, Archives of Acoustics 37, 317 (2012).

[18] W. Batko, J. Felis, A. Flach, T. Kamisiński, T. Giesko, A. Zbrowski, Arch. Acoust. 33, 201 (2008). 